\title{
FABry Disease Patient-Reported Outcome-Gastrolntestinal (FABPRO-GI): A new Fabry disease-specific gastrointestinal outcomes instrument
}

\author{
Alan L. Shields ${ }^{1} \cdot$ Roger E. Lamoureux ${ }^{1} \cdot$ Fiona Taylor $^{1} \cdot$ Jay A. Barth ${ }^{2} \cdot$ Andrew E. Mulberg $^{2} \cdot$ Vivian Kessler $^{2}$. \\ Nina Skuban ${ }^{2}$
}

Accepted: 12 April 2021 / Published online: 29 April 2021

(c) The Author(s) 2021

\begin{abstract}
Purpose Fabry disease is a rare multisystemic disorder caused by functional deficiency of the lysosomal enzyme alphagalactosidase A. Gastrointestinal (GI) signs and symptoms are among the earliest clinical manifestations in patients with Fabry disease but are often nonspecific, misdiagnosed, and untreated. No instruments have been developed specifically to assess GI signs and symptoms in Fabry disease. The FABry disease Patient-Reported Outcome-GastroIntestinal (FABPROGI) was developed to address this unmet need and is intended for use in clinical trials (24-h FABPRO-GI) and real-world settings (7-day FABPRO-GI).

Methods Findings from a literature review, expert advisory meetings, and patient concept elicitation interviews (CEIs) were summarized into conceptual models. These conceptual models were used to develop preliminary versions of the 24-h and 7-day FABPRO-GI. Cognitive debriefing interviews (CDIs) were conducted with additional patients to assess content validity, including understandability, relevance, and comprehensiveness of the preliminary versions of the 24-h and 7-day FABPRO-GI.

Results Literature review ( $n=17$ articles), expert advisory meetings $(n=5)$, and patient CEIs $(n=17)$ identified mostly overlapping Fabry disease-related GI signs and symptoms, including abdominal cramps, bloating, and diarrhea, and informed development of the preliminary 24-h and 7-day FABPRO-GI. CDIs $(n=15)$ provided evidence of content validity and informed revisions of the 24-h and 7-day FABPRO-GI.

Conclusion With evidence of content validity, the 24-h and 7-day FABPRO-GI are the first Fabry disease-specific patientreported outcomes to assess GI signs and symptoms in patients with Fabry disease with potential for use in clinical trials and real-world settings, respectively.
\end{abstract}

Keywords Fabry disease $\cdot$ FABPRO-GI $\cdot$ Patient-reported outcomes $\cdot$ Gastrointestinal signs and symptoms

Abbreviations
$\begin{array}{ll}\alpha \text {-Gal A } & \alpha \text {-Galactosidase A } \\ \text { CDI } & \text { Cognitive debriefing interviews } \\ \text { CEI } & \text { Concept elicitation interviews } \\ \text { ERT } & \text { Enzyme replacement therapy } \\ \text { FABPRO-GI } & \text { FABry Disease Patient-Reported } \\ & \text { Outcome-GastroIntestinal } \\ \text { Gastrointestinal }\end{array}$
GI
$\begin{array}{ll}\begin{array}{l}\text { Alan L. Shields } \\ \text { Alan.Shields @adelphivalues.com }\end{array} \\ \text { Adelphi Values, 290 Congress Street, 6th Floor, Boston, } \\ \text { MA 02210, USA } \\ \text { Amicus Therapeutics, Inc., Cranbury, NJ, USA }\end{array}$

GSRS Gastrointestinal Symptom Rating Scale

NFDD National Fabry Disease Foundation

PROs Patient-reported outcomes

\section{Plain English Summary}

Fabry disease is a rare disorder caused by the deficiency of a specific enzyme called alpha-galactosidase A, which is required for the proper function of most cells throughout the body. Although gastrointestinal (GI) signs and symptoms are common in patients with Fabry disease and often appear early in the course of the disease, they are often misdiagnosed or untreated. No instruments have been developed to properly assess these GI signs and symptoms in patients with 
Fabry disease. This study attempted to develop assessment tools to effectively evaluate GI signs and symptoms in this patient population in clinical trial and real-world settings. Using information obtained from a literature search, expert advisory meetings and patient interviews, investigators were able to identify a set of GI signs and symptoms to include in a 24-h and a 7-day FABry disease Patient-Reported Outcome-GastroIntestinal (FABPRO-GI) questionnaire for use in clinical trials and real-world settings, respectively. These questionnaires may aid early diagnosis and monitoring of treatment effects in Fabry disease.

\section{Introduction}

Fabry disease is an $\mathrm{X}$-linked multisystemic disorder caused by pathogenic GLA variants that result in functional deficiency of the lysosomal enzyme $\alpha$-galactosidase A ( $\alpha$-Gal A) $[1,2]$. Progressive accumulation of globotriaosylceramide and related glycosphingolipids can be detected in most cell types and visceral tissues [1,2]. Glycosphingolipid accumulation can promote several pathological processes including the activation of Toll-like receptors, triggering inflammation and fibrosis cascades [3-5], and lead to a broad range of manifestations including gastrointestinal (GI) signs and symptoms, neuropathic pain, cardiomyopathy, stroke, and renal insufficiency $[1,6]$.

GI complaints are among the earliest and most frequent general complaints in patients with Fabry disease, affecting more than $50 \%$ of all patients with women being slightly more affected than men [7-11]. More specifically, abdominal pain was reported by $28 \%$ of adult patients and $49 \%$ of pediatric patients enrolled in the Fabry Outcome Survey [7] and by $21 \%$ of females and $13 \%$ of males enrolled in the Fabry Registry [12]. In addition, diarrhea, often associated with significant urgency and frequency [13], was reported in $19 \%$ of adult patients and $25 \%$ of pediatric patients from the Fabry Outcome Survey [7] and 19\% of females and 12\% of males enrolled in the Fabry Registry [12].

Despite their negative impact on the patient's healthrelated quality of life, GI signs and symptoms are nonspecific and often overlooked in the management of Fabry disease [11]. In a study of 108 adult patients with Fabry disease enrolled in the Fabry Outcome Survey, healthrelated quality of life as assessed by EQ-5D questionnaire was significantly lower in patients with GI signs and symptoms compared with patients without GI signs and symptoms [7]. Many patients experience nonspecific Fabry disease-related GI signs and symptoms that resemble those of GI disorders and are prone to be misdiagnosed $[14,15]$. For example, a study of 33 patients with Fabry disease and GI signs and symptoms showed that $16 / 25$ adult patients and $2 / 8$ pediatric patients exhibited GI signs and symptoms that resembled those of functional GI disorders [14].

Improvements in GI signs and symptoms have been reported in patients receiving migalastat, a small molecule pharmacological chaperone, or enzyme-replacement therapy (ERT) with recombinant human $\alpha-G a l$ A [20-22]. In addition, patients receiving migalastat demonstrated improvement in the only randomized controlled trial conducted with a validated GI instrument [23]. The effect of migalastat on GI signs and symptoms was assessed in ERT-naive patients from the phase 3 FACETS study using the Gastrointestinal Symptom Rating Scale (GSRS) $[21,23]$. Following 6 months of migalastat treatment, a significantly greater proportion of patients receiving migalastat achieved clinically meaningful improvement in the GSRS diarrhea domain compared with placebo $(P=0.02)$ [23]. Infusion with agalsidase alfa was associated with a statistically significant reduction $(14 \%$; $P<0.05)$ from baseline in the prevalence of abdominal pain in a study of 58 patients from the Fabry Outcome Survey [7]. Similarly, postprandial pain, nausea, and vomiting substantially improved from baseline following 24 weeks of agalsidase beta treatment in an open-label study of 16 pediatric patients [24].

Although these studies provide evidence of the effect of ERT and migalastat treatment on GI signs and symptoms, none of the survey instruments used were developed for patients with Fabry disease or validated for use in this patient population, including the GSRS [21]. This observation suggests the need for a content-valid, psychometrically sound, and clinically interpretable Fabry diseasespecific instrument to assess GI signs and symptoms. This report describes the development of the FABry disease Patient-Reported Outcome-Gastro Intestinal (FABPROGI) to assess Fabry disease-related GI signs and symptoms. Given that patient-reported outcomes (PROs) can provide value outside clinical trials for aspects of disease management such as treatment monitoring in clinical practice [25], PROs have been developed for use in multiple settings [26]. For this purpose, 2 versions of the FABPROGI were developed: the 24-h FABPRO-GI for clinical trials and the 7-Day FABPRO-GI for real-world settings.

\section{Methods}

Consistent with measurement best practices [27] and regulatory guidance on the development of PROs [28], the 24-h and 7-day FABPRO-GI were developed based on results from a comprehensive literature review, expert advisory meetings, and patient concept elicitation interviews (CEIs) (Fig. 1). 


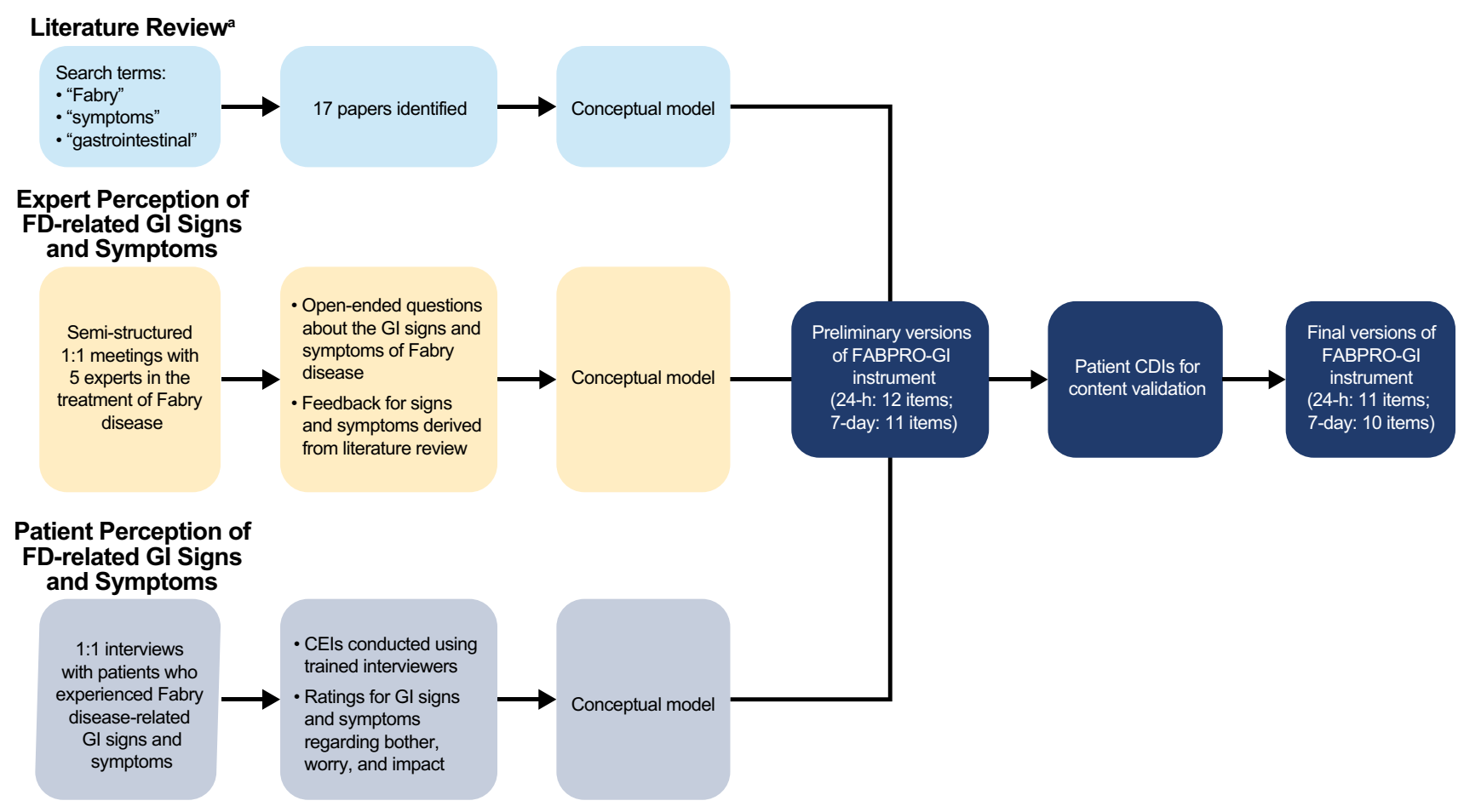

Fig. 1 FABPRO-GI development process. $C D I$ cognitive debriefing interview, $C E I$ concept elicitation interviews, FABPRO-GI FABry disease Patient-Reported Outcome-Gastrointestinal, GI gastrointes-

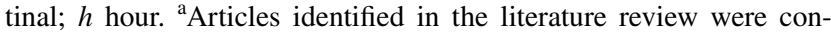
sidered relevant if they focused primarily on the GI symptoms of

\section{Literature Review and Expert Advisory Meetings}

MEDLINE, Embase, and PsycINFO were searched using the OvidSP platform for the time period 2005-July 1, 2015 to identify peer-reviewed articles relating to GI signs and symptoms of Fabry disease. Search terms were "Fabry," "symptoms," and "gastrointestinal." Abstracts were screened to identify relevant articles, and additional references were identified by manually inspecting the reference lists of relevant articles from the search as well as targeted searches of the National Fabry Disease Foundation (NFDF) patient advocacy website (https://www.fabrydisease.org/). Relevant articles primarily focusing on the GI signs and symptoms of Fabry disease were identified, and articles were excluded if they primarily focused on the pathogenesis, genetics, or molecular histology of Fabry disease; primarily focused on non-GI signs and symptoms of Fabry disease; or solely discussed Fabry disease in a population aged $<16$ years.

Semi-structured 1:1 meetings with experts experienced in the treatment of Fabry disease were conducted via telephone by trained interviewers between December 2015 and April 2016. Meetings were designed to give the experts an opportunity to spontaneously describe GI sign- and symptomlevel concepts by asking them open-ended questions about the GI signs and symptoms of Fabry disease. In addition,
Fabry disease. Articles were excluded if they focused primarily on the pathogenesis, genetics, or molecular histology of Fabry disease; focused primarily on non-GI symptoms of Fabry disease; or discussed solely Fabry disease in a population $<16$ years of age

descriptive data were collected relating to the geographic location, years of experience treating patients with Fabry disease, medical specialty, approximate number of patients with Fabry disease seen in a typical month, and work setting.

\section{Patient Perspective}

\section{Patients}

One-on-one interviews were conducted with patients with Fabry disease. Patients were recruited between September and November 2015 through the NFDF email list. Eligible patients were fluent in English, aged $\geq 16$ years, and selfreported having received a diagnosis of Fabry disease and having $\geq 1$ GI sign or symptom (eg, diarrhea, constipation, abdominal pain) in the 14 days prior to study entry.

\section{Patient interviews}

Semi-structured 1:1 CEIs with patients with Fabry disease were conducted in person or via telephone by trained interviewers. Interviewers recorded patient expressions of Fabry disease-related GI signs or symptoms including overall impression, severity, occurrence, frequency, duration, and changes over time. Patients were asked to rate 
each current GI sign or symptom with respect to the level of bother, worry, and impact it caused on a scale of 0 (no bother/worry/impact) to 10 (most bothersome/worrisome/ impactful). Patients were also asked to report the 5 signs or symptoms they would most like to see improve if an effective treatment was available to them.

\section{Development of Conceptual Models}

Concepts were organized into and presented in conceptual models defined as heuristic classification schemes linking a disease state to its proximal and increasingly distal health outcomes [29]. Conceptual models were developed separately based on Fabry disease-related GI signs and symptoms from the literature review, expert advisory meetings, and CEIs (patient interviews) (Fig. 1). Experts were asked to review and provide feedback on the Fabry disease-related GI sign and symptom conceptual model derived from the literature review.

\section{Development of the 24-h and 7-day FABPRO-GI}

The preliminary versions of the 24-h and 7-day FABPRO-GI were drafted based on results consolidated from conceptual models for the literature review, expert advisory meetings, and patient CEIs according to measurement best practices [27] and regulatory guidance [28] (Fig. 1). Items assessing signs or symptoms asked patients to rate the severity of the specified sign or symptom "at its worst" in the $24 \mathrm{~h}$ (24-h FABPRO-GI) or 7 days (7-day FABPRO-GI) prior to assessment on an 11-point (0-10) numeric rating scale. The preliminary versions of the 24-h and 7-day FABPRO-GI were then subject to cognitive debriefing interviews (CDIs) for readability and patient comprehension, and results informed the final versions (Fig. 1).

Patients $(n=15)$ participating in CDIs were recruited in September 2016 through the NFDF and met the same eligibility criteria as patients participating in the CEIs. CDIs were conducted in person by a trained interviewer using a semi-structured CDI guide and consisted of 2 stages: a "think-aloud" stage in which the patient completed the questionnaire while verbalizing the process (without any input from the interviewer) and a discussion stage, in which patients reported whether the instructions, items, response options, and recall periods were relevant and appropriate for the questionnaires. Patients were also asked if they would suggest the questionnaires be revised to make them more relevant, comprehensive, and interpretable. CDIs were then transcribed, coded, and analyzed to determine whether revisions to the 24-h and 7-day FABPRO-GI were necessary.

\section{Data Handling and Analysis}

Transcribed meetings and interviews were anonymized, coded, and analyzed using descriptive statistics and qualitative analytic methods. The transcripts were entered into ATLAS.ti Version 7.5.6 (ATLAS.ti Scientific Software Development GmbH; Berlin, Germany) and coded by researchers by linking transcript text to a code from the codebook that best characterized the concept. For CEIs, each code contained information about the domain (eg, symptom), root concept (eg, abdominal pain), and descriptor (eg, severity, frequency, duration, or location). For CDIs, each code contained information about interpretation of questionnaire instructions, items, and response options, experience of the symptom being assessed, and suggestions for revision. Development of the codebook, a comprehensive list of all codes used to characterize important segments of transcript text, was an iterative process in which codes were added, deleted, or merged as each interview transcript became available. For CEIs, saturation, the point at which no new information is gained from additional interviewees, was evaluated in a stepwise process.

The frequency of experts who indicated that a given sign or symptom concept was part of the Fabry disease-related GI experience for patients was determined by counting the number of unique experts who mentioned the concept at least once during the expert advisory meeting. The frequency of experts who considered each concept to be an important treatment outcome was also determined. Furthermore, concept frequency was determined for patients with Fabry disease, as well as a qualitative analysis of bother, worry, and impact rating data. Data characterizing the study population of experts and patients with Fabry disease were summarized descriptively.

\section{Results}

\section{Literature review}

Seventeen relevant articles were identified from the literature search (Online Resource Figure S1), including 9 primary research articles, 7 review articles, and 1 resource document from the NFDF website. Review of these sources identified 14 Fabry-related GI signs and symptoms (in decreasing order of frequency): diarrhea, abdominal pain, nausea, vomiting, bloating, constipation, weight loss, abdominal cramping, chest pain (non-cardiac, presumed esophageal origin), excessive belching, feeling of satiety, gas, indigestion, and reflux. Concepts relating to GI signs and symptoms of Fabry disease were subsequently organized into a literature-based conceptual model. 


\section{Expert Perspectives}

Five experts from the United States $(n=3)$ and England $(n=2)$ participated in advisory meetings. These individuals worked in a variety of clinical settings, had treated patients with Fabry disease for between 15 and 24 years, and reported seeing approximately 6-80 patients with Fabry disease per month. Together, these experts reported a total of 12 GI signs and symptoms of Fabry disease: diarrhea, abdominal pain, early satiety, abdominal cramps, constipation, gas (flatulence), nausea, vomiting, poor appetite, acid reflux, bloating, and weight loss (Fig. 2A). The 3 signs and symptoms reported by most experts (diarrhea $[n=5 ; 100 \%]$; abdominal pain $[n=4 ; 80 \%]$; and early satiety $[n=3 ; 80 \%]$ ) were also identified by these experts as important treatment outcomes from their perspective and/or the patients' perspective ( $n=2 ; n=2$; and $n=1$, respectively) (Fig. 2B). Other treatment outcomes deemed as important for patients with Fabry disease by the experts were related to constipation $(n=2 ; 40 \%)$, asymptomatic status $(n=1 ; 20 \%)$, and less GI sign or symptom interference in the patient's life $(n=1 ; 20 \%)$. Upon reviewing the literature-based conceptual model, 1 expert agreed with all GI signs and symptoms in the model and $3(60 \%)$ experts suggested removing $\geq 1$ sign or symptom (reflux $[n=2]$; weight loss $[n=2]$; chest pain $[n=1]$; indigestion $[n=1]$ ).

Four experts believed GI signs and symptoms of Fabry disease varied with patient age, and all experts agreed that the presentation of GI signs and symptoms in Fabry disease varied with patient sex. Regarding treatment of these signs and symptoms, experts confirmed that no Fabry

\section{A Expert-reported Signs and Symptoms}

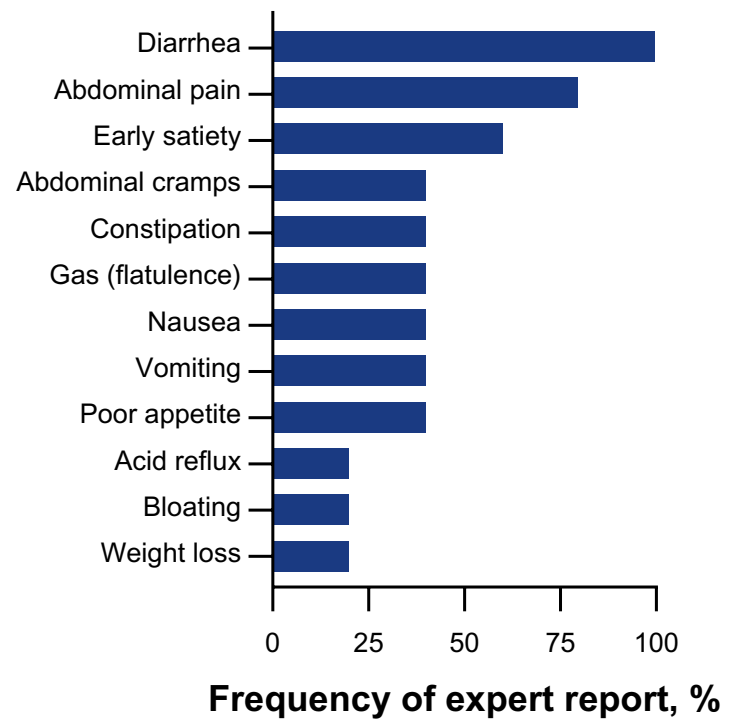

disease-specific treatment was available to them to prescribe to their patients and, instead, they relied upon treatments used for other GI signs or symptoms including medications such as antacids, antispasmodic drugs, loperamide, and metoclopramide, as well as dietary changes.

\section{Patient Perspectives}

Nineteen patients were interviewed and 17 were included in the analysis ( 2 patients were excluded based on a diagnosis of gastroparesis reported during the CEI; Table 1). Among patients contributing to the analysis, 10 patients $(58.8 \%)$ were female, the mean (range) age was 33.7 (16.7-60.8) years, and 12 patients $(70.6 \%)$ were currently receiving ERT. Most patients self-reported the severity of their Fabry disease-related GI signs and symptoms as moderate $(n=$ $11 ; 64.7 \%)$ or mild $(n=3 ; 17.6 \%)$. To determine whether an adequate sample size had been achieved for identifying GI signs and symptoms, a concept saturation grid was developed, which showed elicitation of $92.3 \%$ of all concepts in the first $75 \%$ of interviews, supporting the achievement of saturation (Online Resource Table S1).

Of the Fabry disease-related GI signs and symptoms mentioned by patients during the CEIs, the most commonly reported (by $\geq 50 \%$ of patients) were diarrhea (by $n=13 ; 76.5 \%)$, bloating ( $n=10 ; 58.8 \%)$, constipation ( $n=10 ; 58.8 \%)$, and cramping $(n=9 ; 52.9 \%)$ (Fig. 3 ). For the signs and symptoms reported by $\geq 50 \%$ of patients, the reported sign or symptom severity ranged from mild $(n=1$ of 7) to severe ( $n=4$ of 7$)$ for diarrhea, and most patients reported their bloating as mild to moderate $(n=7$ of 8$)$, their

\section{B Important Treatment Outcomes}

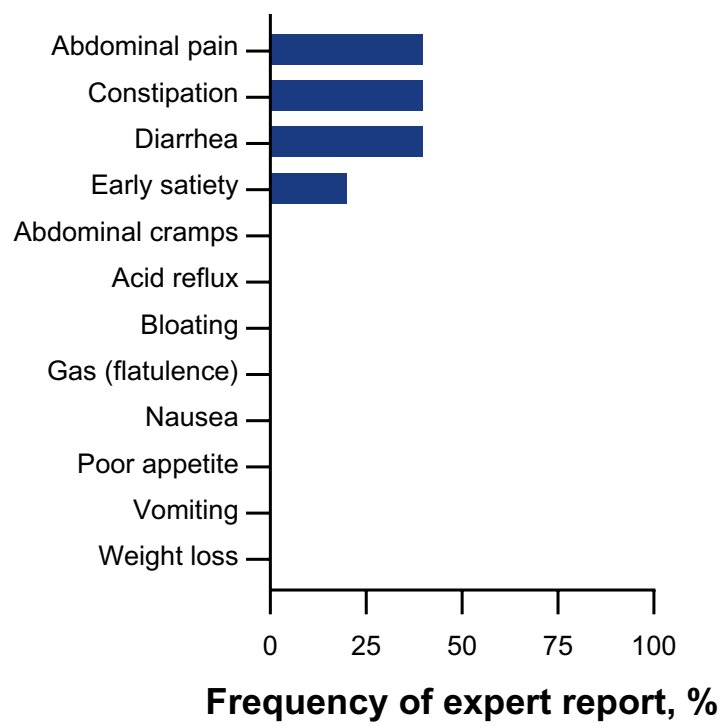

Fig. 2 Expert-reported (A) GI signs and symptoms and (B) important treatment outcomes in Fabry disease. GI gastrointestinal 
Table 1 Demographics and disease characteristics of patients with Fabry disease participating in CEIs and CDIs

\begin{tabular}{|c|c|c|}
\hline & CEIs $(N=17)$ & CDIs $(N=15)$ \\
\hline Age, years, mean (SD) & $33.7(14.2)$ & $39.6(16.7)$ \\
\hline Female, n (\%) & $10(58.8)$ & $11(73.3)$ \\
\hline \multicolumn{3}{|l|}{ Race, n (\%) } \\
\hline White/Caucasian & $11(64.7)$ & $13(86.7)$ \\
\hline Black or African American & - & $2(13.3)$ \\
\hline Other & $5(29.4)$ & \\
\hline Not answered & $1(5.9)$ & \\
\hline \multicolumn{3}{|l|}{ Highest level of education, $\mathrm{n}(\%)$} \\
\hline High school diploma (or GED) or less & $6(35.3)$ & $7(46.7)$ \\
\hline Some college or certificate program & $4(23.5)$ & $3(20.0)$ \\
\hline College or university degree (2- or 4-year) & $5(29.4)$ & $3(20.0)$ \\
\hline Graduate degree & $1(5.9)$ & $2(13.3)$ \\
\hline Other & $1(5.9)^{\mathrm{a}}$ & - \\
\hline Time since diagnosis, years, mean (SD) & $11.8(12.2)$ & $7.9(6.2)$ \\
\hline \multicolumn{3}{|l|}{ Health status, $\mathrm{n}(\%)^{\mathrm{b}}$} \\
\hline Excellent & $1(5.9)$ & - \\
\hline Very good & $6(35.3)$ & $2(13.3)$ \\
\hline Good & $6(35.3)$ & $9(60.0)$ \\
\hline Fair & $3(17.6)$ & $4(26.7)$ \\
\hline Poor & $1(5.9)$ & - \\
\hline \multicolumn{3}{|l|}{ Fabry disease-related GI symptom severity, $\mathrm{n}(\%)^{\mathrm{b}}$} \\
\hline Very mild & $1(5.9)$ & $1(6.7)$ \\
\hline Mild & $3(17.6)$ & $2(13.3)$ \\
\hline Moderate & $11(64.7)$ & $11(73.3)$ \\
\hline Severe & $1(5.9)$ & $1(6.7)$ \\
\hline Very severe & $1(5.9)$ & - \\
\hline \multicolumn{3}{|l|}{ Current medications, $\mathrm{n}(\%)^{\mathrm{b}, \mathrm{c}, \mathrm{d}}$} \\
\hline Prophylactic pain agents ${ }^{\mathrm{e}}$ & $6(35.3)$ & $4(26.7)$ \\
\hline NSAIDs & $5(29.4)$ & $3(20.0)$ \\
\hline Analgesics $^{\mathrm{f}}$ & $5(29.4)$ & $5(33.3)$ \\
\hline Anti-nausea agents & $3(17.6)$ & $3(20.0)$ \\
\hline Antacids & $3(17.6)$ & $4(26.7)$ \\
\hline Docusate & $2(11.8)$ & $2(13.3)$ \\
\hline Enemas & $2(11.8)$ & - \\
\hline Prokinetics & $2(11.8)$ & $3(20.0)$ \\
\hline Anti-diarrheal agents & $1(5.9)$ & $1(6.7)$ \\
\hline Bismuth & $1(5.9)$ & $2(13.3)$ \\
\hline GI disturbance medication & $1(5.9)$ & $4(26.7)$ \\
\hline Anti-spasmodic agents & - & $1(6.7)$ \\
\hline Other & $2(11.8)$ & $5(33.3)$ \\
\hline \multicolumn{3}{|l|}{ Abdominal surgeries, $\mathrm{n}(\%)^{\mathrm{b}, \mathrm{c}}$} \\
\hline Hysterectomy, n (\%) & $3(17.6)$ & $3(20.0)$ \\
\hline Removal of appendix & $2(11.8)$ & $3(20.0)$ \\
\hline Removal of gallbladder & $1(5.9)$ & $4(26.7)$ \\
\hline
\end{tabular}

$C D I$ cognitive debriefing interview, $C E I$ concept elicitation interview, $G E D$ general educational development, $G I$ gastrointestinal, NSAID nonsteroidal anti-inflammatory drugs, $S D$ standard deviation

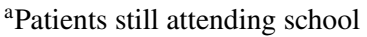

${ }^{\mathrm{b}}$ Patient-reported

${ }^{\mathrm{c}}$ Not mutually exclusive

${ }^{\mathrm{d}}$ Enzyme replacement therapy was excluded

${ }^{\mathrm{e}}$ For example, phenytoin, carbamazepine, or gabapentin

${ }^{\mathrm{f}}$ For example, narcotics, opioids such as codeine, hydrocodone, Demerol, OxyContin, or Percocet 


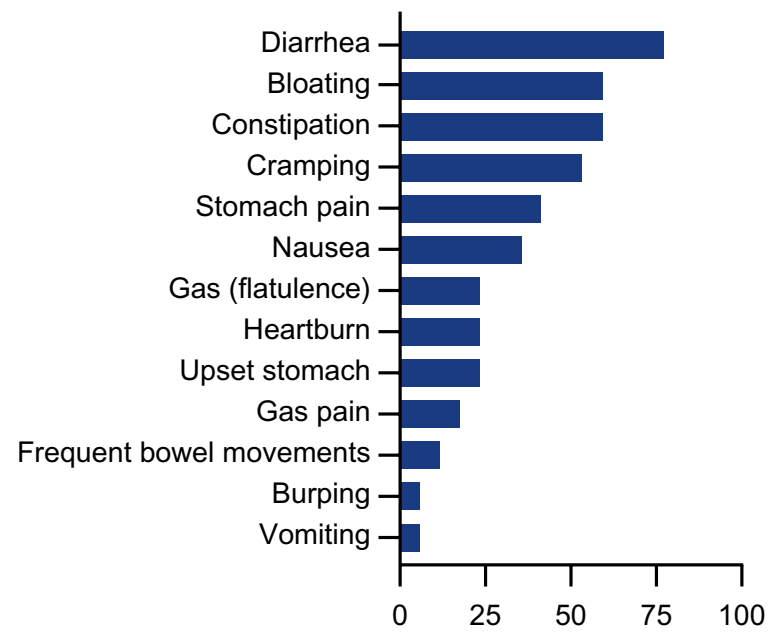

Frequency of patient report, \%

Fig. 3 Patient-reported GI signs and symptoms in Fabry disease. GI gastrointestinal

constipation as severe ( $n=6$ of 9 ), and their cramping as "intense," "severe," or "very painful" $(n=6$ of 8$)$.

Patient perception of the bother, worry, and impact of each GI sign or symptom was rated on a scale of 0 to 10, where higher scores indicate more bother, worry, or impact, and is reported in Table 2. Among the GI signs and symptoms reported by $\geq 50 \%$ of patients (diarrhea, bloating, constipation, and cramping), mean bother ratings ranged from 4.9 to 6.5 , mean worry ratings ranged from 3.5 to 5.5 , and mean impact ratings ranged from 3.3 to 6.0. Diarrhea was the symptom most frequently ranked as the most important to improve $(n=6)$ and was most frequently ranked among the top 5 signs and symptoms $(n=11)$, followed by bloating $(n=9)$ and constipation $(n=8)$. The 13 Fabry disease-related GI sign and symptom concepts reported by patients during the CEIs were organized into a patient-centric conceptual model.

\section{Development of the 24-h and 7-day FABPRO-GI}

Three conceptual models were derived from the literature review, expert advisory meetings, and patient CEIs, which showed largely overlapping GI signs and symptoms (Fig. 4). The signs and symptoms derived from the conceptual models informed the target concepts of interest (bloating; stomach pain; cramping; nausea; acid reflux; heartburn; indigestion; constipation; severity, frequency, and consistency of diarrhea; and bowel movement frequency), and 2 preliminary FABPRO-GI questionnaires were developed to assess those concepts (1) in the $24 \mathrm{~h}$ prior to assessment during clinical trials (Table 3 ) and (2) in the 7 days prior to assessment in real-world settings (Table 4). The concepts assessed by the 7-day FABPRO-GI are the same as those assessed
Table 2 Patient-reported GI signs and symptoms and their bother, worry, and impact

\begin{tabular}{|c|c|c|c|c|c|c|c|}
\hline \multirow[t]{2}{*}{ Concept $^{\mathrm{a}}$} & \multirow[t]{2}{*}{ Ratings/Patients ${ }^{\mathrm{b}}$} & \multicolumn{2}{|c|}{ Bother rating $^{\mathrm{c}}$} & \multicolumn{2}{|c|}{ Worry rating $^{\mathrm{c}}$} & \multicolumn{2}{|c|}{ Impact rating $^{\mathrm{c}}$} \\
\hline & & $n$ & Mean (SD) & $n$ & Mean (SD) & $\mathrm{n}$ & Mean (SD) \\
\hline Diarrhea $(n=13)$ & $11 / 13$ & 11 & $6.4(2.0)$ & 11 & $3.5(2.9)$ & 11 & $5.1(3.3)$ \\
\hline Bloating $(n=10)$ & $9 / 10$ & 9 & $4.9(2.3)$ & 9 & $3.6(3.2)$ & 9 & $3.3(3.3)$ \\
\hline Constipation $(n=10)$ & $9 / 10$ & 9 & $6.4(3.0)$ & 9 & $5.5(3.3)$ & 9 & $5.8(2.8)$ \\
\hline Cramping $(n=9)$ & $8 / 9$ & 8 & $6.5(2.3)$ & 8 & $4.4(2.7)$ & 7 & $6.0(3.4)$ \\
\hline Stomach pain $(n=7)$ & $7 / 7$ & 7 & $6.1(2.9)$ & 7 & $4.9(1.7)$ & 7 & $5.0(2.9)$ \\
\hline Nausea $(n=6)$ & $5 / 6$ & 5 & $6.2(2.8)$ & 5 & $2.4(1.9)$ & 5 & $4.0(3.5)$ \\
\hline Gas $(n=4)$ & $4 / 4$ & 4 & $3.8(3.3)$ & 4 & $4.5(5.3)$ & 4 & $5.0(4.2)$ \\
\hline Heartburn $(n=4)$ & $4 / 4$ & 4 & $5.5(3.7)$ & 4 & $4.5(3.8)$ & 4 & $3.8(1.7)$ \\
\hline Upset stomach $(n=4)$ & $3 / 4$ & 3 & $5.7(2.1)$ & 3 & $3.0(2.6)$ & 3 & $5.0(4.4)$ \\
\hline Gas pain $(n=3)$ & $2 / 3$ & 2 & $6.5(0.7)$ & 2 & $3.0(4.2)$ & 2 & $4.5(4.9)$ \\
\hline $\begin{array}{l}\text { Frequent bowel move- } \\
\text { ments }(n=2)\end{array}$ & $1 / 2$ & 1 & 7.0 & 1 & 8.0 & 1 & 6.0 \\
\hline Burping $(n=1)$ & $0 / 1$ & 0 & - & 0 & - & 0 & - \\
\hline Vomiting $(n=1)$ & $1 / 1$ & 1 & 8.0 & 1 & 4.0 & 1 & 5.0 \\
\hline
\end{tabular}

CEI concept elicitation interview, GI gastrointestinal, $S D$ standard deviation

${ }^{\text {a }}$ Based on the CEIs

${ }^{b}$ Indicates the number of patients who provided a symptom rating out of the total number of patients reported experiencing the symptom during the open-ended discussion. The number of patients who had the opportunity to provide a rating for the symptom may be less than the total frequency count for some symptoms as patients were only able to rate symptoms that the interviewer specifically asked about during the interview, prior to the complete analysis of qualitative data

${ }^{c}$ Rated on a $0-10$ scale where a higher score indicates more bother, worry, or impact 
by the 24-h FABPRO-GI, with the difference that the 7-day FABPRO-GI uses a recall period of "the past 7 days" and asks subjects to report the average severity of symptoms over that period.

To evaluate the preliminary content of the 24-h and 7-day FABPRO-GI, 15 patients (Table 1) with Fabry disease participated in CDIs to provide feedback on all instructions, items, and response options of the 24-h FABPRO-GI, and the instructions and Items 2 (stomach pain), 8 (constipation), and 11 (diarrhea severity) of the 7-day FABPRO-GI. Eleven patients $(73.3 \%$ ) were female, the mean (range) age was 39.6 (16.9-64.0) years, and the majority $(n=11 ; 73.3 \%)$ reported their Fabry disease-related GI sign and symptom severity as moderate (Table 1).

For the 24-h FABPRO-GI questionnaire, all but 1 patient who provided an interpretable response $(n=13 ; 92.9 \%)$ interpreted the instructions as intended. The patient who did not interpret a portion of the instructions as intended showed some confusion at first but proceeded to answer items as intended. In addition, most patients $(n=14 ; 93.3 \%)$ understood the 24-h recall period within the instructions. All 13 patients who provided an interpretable response interpreted the instructions of the 7-day FABPRO-GI as intended, and all patients $(n=15)$ understood the 7-day recall period.

Overall, patients interpreted all items of the 24-h and 7-day FABPRO-GI questionnaires as intended. It is notable that 2 patients (13.3\%) did not interpret Item 7 (indigestion) of the 24-h FABPRO-GI as intended, and even those who interpreted Item 7 as intended were thinking about a wide variety of different symptoms, many of which overlapped with concepts already in the questionnaire. For Items 1-10 and 12 , at least $92.3 \%$ of patients reported experiencing the sign or symptom either within or prior to the 24-h recall period used in the questionnaire, whereas Item 11 (diarrhea consistency) applied only to 4 patients $(26.7 \%)$ as they were the only patients who reported $\geq 1$ episode of diarrhea within the past $24 \mathrm{~h}$. All patients interpreted the response scales of the 24-h FABPRO-GI as intended by the developers. In addition, all patients stated the questionnaire was easy to complete. Two patients (14.3\%) indicated 1 or more items were repetitive, 4 patients (26.7\%) suggested deleting one or more items, and the items most frequently ranked as the
Fig. 4 Fabry disease-related GI sign and symptom concepts as reported in the literature, by experts, and by patients. GI gastrointestinal

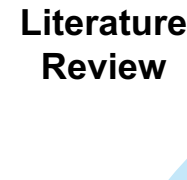
Expert Advisory Meetings $(\mathrm{N}=5)$

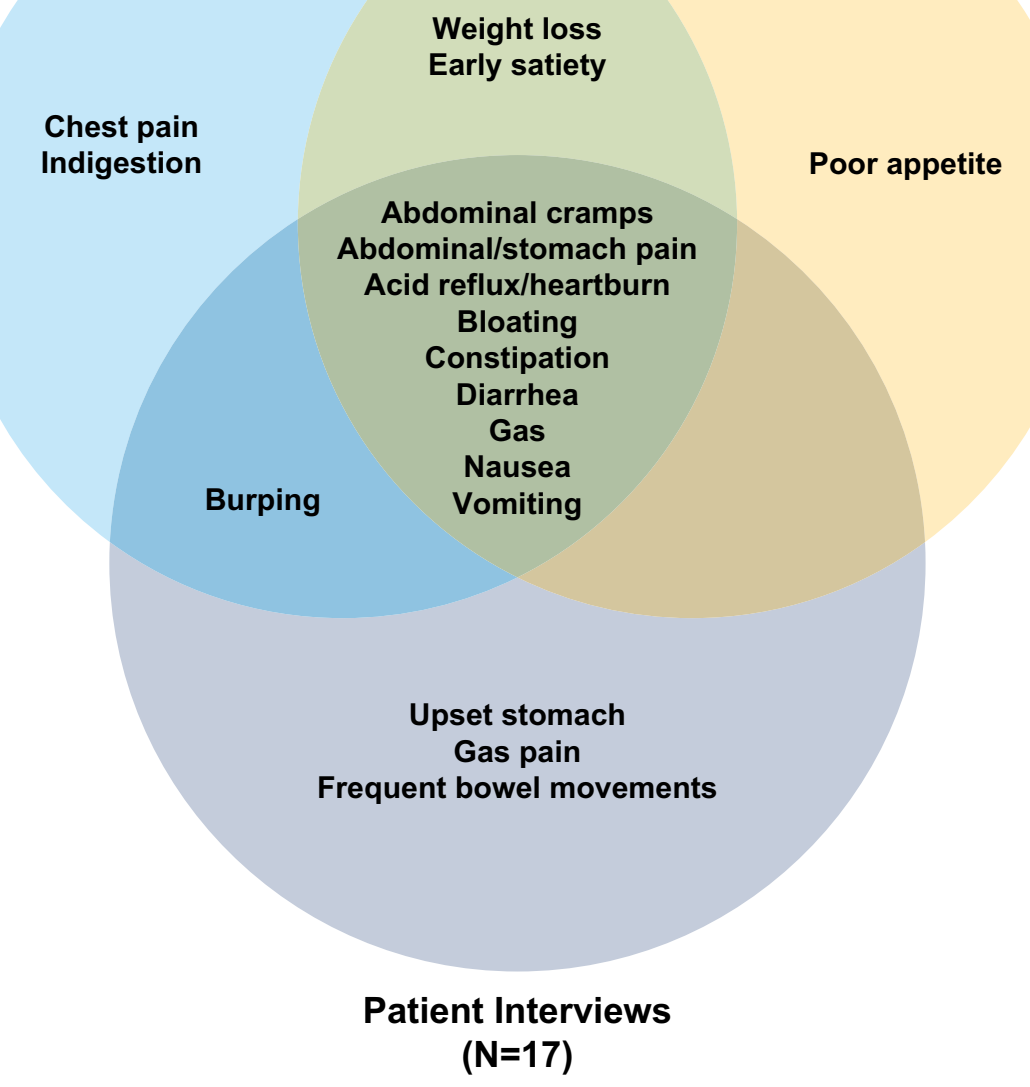


Table 3 Items on the Preliminary 24-h FABPRO-GI

\begin{tabular}{|c|c|}
\hline Domain & Item \\
\hline Disease-related GI sign and symptom severity & $\begin{array}{l}\text { 1. Over the past } 24 \mathrm{~h} \text {, how severe was your worst bloating? } \\
\text { 2. Over the past } 24 \mathrm{~h} \text {, how severe was your worst stomach pain? } \\
\text { 3. Over the past } 24 \mathrm{~h} \text {, how severe was your worst cramping? } \\
\text { 4. Over the past } 24 \mathrm{~h} \text {, how severe was your worst nausea? } \\
\text { 5. Over the past } 24 \mathrm{~h} \text {, how severe was your worst acid reflux? } \\
\text { 6. Over the past } 24 \mathrm{~h} \text {, how severe was your worst heartburn? } \\
\text { 7. Over the past } 24 \mathrm{~h} \text {, how severe was your worst indigestion? } \\
\text { 8. Over the past } 24 \mathrm{~h} \text {, how severe was your worst constipation? } \\
\text { 12. Over the past } 24 \mathrm{~h} \text {, how severe was your worst diarrhea? }\end{array}$ \\
\hline Frequency of bowel movements & 9. Over the past $24 \mathrm{~h}$, how many bowel movements did you have? \\
\hline Frequency of diarrhea & 10. Over the past $24 \mathrm{~h}$, how many times did you have diarrhea? \\
\hline Consistency of diarrhea & 11. Over the past $24 \mathrm{~h}$, what was the consistency of your worst diarrhea? \\
\hline
\end{tabular}

The bolded text is as it appears on the questionnaire to emphasize the rating is for "worst" ( 24 h FABPRO-GI) and "average" (7-day FABPROGI) symptoms, respectively

FABPRO-GI FABry Disease Patient Reported Outcome-Gastrointestinal

aItems were scored on a 0-10 scale, where "0" indicated the absence of the sign or symptom and " 10 " indicated worst possible

${ }^{\mathrm{b}}$ Item 7 was removed from the final 24-h FABPRO-GI

Table 4 Items on the Preliminary 7-day FABPRO-GI ${ }^{\mathrm{a}}$

\begin{tabular}{|c|c|}
\hline Domain & Item \\
\hline Disease-related GI sign and symptom severity & $\begin{array}{l}\text { 1. Over the past } 7 \text { days, how severe was your bloating on average? } \\
\text { 2. Over the past } 7 \text { days, how severe was your stomach pain on average? } \\
\text { 3. Over the past } 7 \text { days, how severe was your cramping on average? } \\
\text { 4. Over the past } 7 \text { days, how severe was your nausea on average? } \\
\text { 5. Over the past } 7 \text { days, how severe was your acid reflux on average? } \\
\text { 6. Over the past } 7 \text { days, how severe was your heartburn on average? } \\
\text { 7. Over the past } 7 \text { days, how severe was your indigestion on average? } \\
\text { 8. Over the past } 7 \text { days, how severe was your constipation on average? } \\
\text { 11. Over the past } 7 \text { days, how severe was your diarrhea on average? }\end{array}$ \\
\hline Frequency of bowel movements & 9. Over the past 7 days, how many bowel movements per day did you have on average? \\
\hline Frequency of diarrhea & 10. Over the past 7 days, how many times per day did you have diarrhea on average? \\
\hline
\end{tabular}

The bolded text is as it appears on the questionnaire to emphasize the rating is for "worst" (24 h FABPRO-GI) and "average" (7-day FABPROGI) symptoms, respectively

FABPRO-GI FABry Disease Patient Reported Outcome-Gastrointestinal

aItems were scored on a $0-10$ scale, where " 0 " indicated the absence of the sign or symptom and " 10 " indicated worst possible

${ }^{\mathrm{b}}$ Item 7 was removed from the final 7-day FABPRO-GI

most important were Items 8 (constipation, $n=8 ; 57.1 \%$ ), Items 10, 11, and 12 (diarrhea frequency/consistency/severity, $n=8 ; 57.1 \%$ ), and Item 2 (stomach pain, $n=2 ; 42.9 \%$ ).

For the 7-day FABPRO-GI, 75\% ( $n=9$ of 12 ), $90.9 \%$ ( $n=8$ of 11$)$, and $90.9 \%(n=8$ of 11$)$ of respondents interpreted Item 2 (stomach pain), Item 8 (constipation), and Item 11 (diarrhea) as intended, respectively. Two patients (16.7\%) assessed severity of their stomach pain based on frequency of occurrence rather than severity. One patient misinterpreted the recall period for each of the 3 items.
On the basis of patient feedback, revisions to the 24-h FABPRO-GI questionnaire included removing Item 7 (indigestion) and the addition of a skip pattern for Items 11 (diarrhea consistency) and 12 (diarrhea severity) for patients who had not experienced diarrhea in the past $24 \mathrm{~h}$. The final 24-h FABPRO-GI consisted of 11 items. Item 7 was also removed from the 7-day FABPRO-GI based on the feedback received to produce the final 10-item questionnaire. 


\section{Discussion}

The results of this study provide evidence of the content validity of the 24-h and 7-day FABPRO-GI in assessing concepts that are relevant to Fabry disease and important to affected patients and that they can do so in ways that patients can easily understand and complete. Informed by the literature, expert advice, and patient input, the 24-h and 7-day FABPRO-GI can be used to assess GI signs and symptoms in patients with Fabry disease. The general understandability, relevance, and comprehensiveness of the 24-h and 7-day FABPRO-GI were evaluated and confirmed in a group of patients aged $\geq 16$ years with Fabry disease with minor modifications to the questionnaires. These were developed in accordance with regulatory guidance [28] and have the potential for use in real-world settings to increase understanding of the GI signs and symptoms experienced by patients with Fabry disease, and in clinical trials to assess the effects of therapy on Fabry disease-related GI signs and symptoms. Moreover, patients with a variety of genotypes were included in this study to represent the broad phenotypic spectrum of Fabry disease.

GI signs and symptoms, often the first clinical manifestation of Fabry disease, are nonspecific, likely contribute to long diagnostic delays and misdiagnoses experienced by patients, and impose substantial psychological stress on patients [11]. During interviews for this study, patients reported experiencing delays and difficulties in obtaining proper diagnosis and treatment. Numerous possible differential diagnoses exist for nonspecific GI signs and symptoms. For example, diarrhea episodes alternating with normal bowel activity or constipation, which may occur in a patient with Fabry disease, may alternatively indicate diarrheapredominant irritable bowel syndrome [11]. Patients with Fabry disease initially presenting with abdominal pain have received wide-ranging misdiagnoses including non-specific pain, food-borne illness, dyspepsia, and gastroesophageal reflux and received treatment for these disorders prior to receiving the correct diagnosis and treatment [15]. Moreover, GI signs and symptoms are associated with psychological stress that may exacerbate pain and discomfort in patients with Fabry disease [11].

To date, no instruments are in clinical use to evaluate Fabry disease-related GI signs and symptoms. Given the nonspecificity of GI signs and symptoms associated with Fabry disease, this may hinder timely diagnosis and highlights the importance of developing a Fabry disease-specific instrument for assessing GI signs and symptoms.

The 24-h and 7-day FABPRO-GI are the first Fabry disease-specific PROs to assess GI signs and symptoms in patients with Fabry disease. Gastrointestinal signs and symptoms in patients with Fabry disease were previously evaluated via patient interviews and instruments designed to assess other gastrointestinal disorders including the GSRS [30, 23] and Rome III criteria [31, 14] (now Rome IV [32]). Given the paucity of PROs developed for Fabry disease, the 24-h and 7-day FABPRO-GI may provide new insights into Fabry disease-related GI signs and symptoms that may facilitate their recognition in patients with Fabry disease participating in clinical trials and those in real-world settings.

The methodology used to develop the 24-h and 7-day FABPRO-GI is similar to those of other recently developed Fabry disease-specific PROs. A self-reported questionnaire to evaluate patient treatment expectations for Fabry disease regarding aspects such as long-term efficacy, impact on daily living, and effective treatment of signs and symptoms was recently developed based on a review of the literature and patient interviews [33]. As content validity and test and retest reliability were demonstrated, this questionnaire was considered to be a suitable instrument for assessing patients with Fabry disease and is currently being used in a phase 4 study [33]. Similarly, a PRO for the evaluation of neuropathic pain in patients with Fabry disease was developed based on CEIs and CDIs for use in pivotal clinical trials [34].

There were several limitations to this study. One is the small number of patients who participated in CEIs $(n=$ $17)$ and CDIs $(n=15)$. However, at least one meta-analytic inquiry across $26 \mathrm{CE}$ studies indicates that small sample sizes have proven adequate for these types of specific inquiries [35]. The consistency of the present saturation results with the referenced meta-analysis enhances confidence in the reliability of conclusions drawn from the research presented here. Second, although this study describes the development and content validation of the 24-h and 7-day FABPRO-GI, evaluation of psychometric performance and score interpretation of these outcome measures in clinical studies is pending.

\section{Conclusions}

The 24-h and 7-day FABPRO-GI can potentially be used in clinical trials and real-world settings, respectively, to monitor and help bring attention to often underrecognized and untreated GI signs and symptoms in patients with Fabry disease.

Supplementary Information The online version contains supplementary material available at https://doi.org/10.1007/s11136-021-02847-9.

Acknowledgements We would like to thank the patients and their families for participation in this study. Third-party medical writing assistance was provided by Lei Bei, $\mathrm{PhD}$, and Stephanie Agbu, PhD, of ApotheCom (Yardley, PA) and was supported by Amicus Therapeutics, Inc. 
Author contributions ALS, FT, JAB, VK, NS, and AEM participated in study design; ALS, REL, FT, and NS assisted in the acquisition of data; ALS, REL, FT, and NS analyzed the data, ALS, REL, FT, JAB, NS, and AEM interpreted the data; ALS, NS, and AEM drafted the article; all authors critically reviewed and revised the article.

Funding This study was funded by Amicus Therapeutics, Inc.

Data availability All data relevant to this study are included in the article or were uploaded as supplementary material.

Code availability Not applicable.

\section{Declarations}

Conflict of interest Alan L. Shields, Roger E. Lamoureux, and Fiona Taylor are employees of Adelphi Values, which provides research services to pharmaceutical drug development companies including Amicus Therapeutics, Inc. Vivian Kessler is an employee of and stockholder in Amicus Therapeutics, Inc. Jay A. Barth, Andrew E. Mulberg, and Nina Skuban are former employees of Amicus Therapeutics, Inc., and hold stock in Amicus Therapeutics, Inc.

Ethical approval Study procedures were approved by the Copernicus Group Independent Review Board (Durham, NC, USA).

Consent to participate All patients provided written informed consent.

Consent for publication All authors provided consent for the publication of this work.

Open Access This article is licensed under a Creative Commons Attribution 4.0 International License, which permits use, sharing, adaptation, distribution and reproduction in any medium or format, as long as you give appropriate credit to the original author(s) and the source, provide a link to the Creative Commons licence, and indicate if changes were made. The images or other third party material in this article are included in the article's Creative Commons licence, unless indicated otherwise in a credit line to the material. If material is not included in the article's Creative Commons licence and your intended use is not permitted by statutory regulation or exceeds the permitted use, you will need to obtain permission directly from the copyright holder. To view a copy of this licence, visit http://creativecommons.org/licenses/by/4.0/.

\section{References}

1. Germain, D. P. (2010). Fabry disease. Orphanet Journal of Rare Diseases, 5, 30

2. Desnick, R. J., Ioannou, Y., \& Eng, C. M. (2016). a-Galactosidase A deficiency: Fabry disease. In: D. Valle, A. L. Beaudet, B. Vogelstein, K. W. Kinzler, E. S. Antonarakis, A. Ballabio, et al., eds. The Online Metabolic and Molecular Bases of Inherited Disease: McGraw-Hill Companies, Inc.

3. Sanchez-Nino, M. D., Carpio, D., Sanz, A. B., Ruiz-Ortega, M., Mezzano, S., \& Ortiz, A. (2015). Lyso-Gb 3 activates Notch1 in human podocytes. Human Molecular Genetics, 24(20), $5720-5732$

4. Anders, H. J., Banas, B., \& Schlondorff, D. (2004). Signaling danger: toll-like receptors and their potential roles in kidney disease. Journal of the American Society of Nephrology, 15(4), 854-867
5. Rozenfeld, P., \& Feriozzi, S. (2017). Contribution of inflammatory pathways to Fabry disease pathogenesis. Molecular Genetics and Metabolism, 122(3), 19-27

6. Schiffmann, R. (2015). Fabry disease. Handbook of Clinical Neurology, 132, 231-248

7. Hoffmann, B., Schwarz, M., Mehta, A., Keshav, S., \& Investigators, F. O. S. E. (2007). Gastrointestinal symptoms in 342 patients with Fabry disease: prevalence and response to enzyme replacement therapy. Clinical Gastroenterology and Hepatology, 5(12), 1447-1453

8. Gold, K. F., Pastores, G. M., Botteman, M. F., Yeh, J. M., Sweeney, S., Aliski, W., et al. (2002). Quality of life of patients with Fabry disease. Quality of Life Research, 11(4), 317-327

9. Mehta, A., Ricci, R., Widmer, U., Dehout, F., Garcia de Lorenzo, A., Kampmann, C., et al. (2004). Fabry disease defined: baseline clinical manifestations of 366 patients in the Fabry Outcome Survey. European Journal of Clinical Investigation, 34(3), 236-242

10. MacDermot, K. D., Holmes, A., \& Miners, A. H. (2001). Anderson-Fabry disease: clinical manifestations and impact of disease in a cohort of 98 hemizygous males. Journal of Medical Genetics, 38(11), 750-760

11. Hilz, M. J., Arbustini, E., Dagna, L., Gasbarrini, A., Goizet, C., Lacombe, D., et al. (2018). Non-specific gastrointestinal features: Could it be Fabry disease? Digestive and Liver Disease, 50(5), 429-437

12. Genzyme Corporation (2007). Fabry RADAR 2007 The Fabry Registry Aggregate Data Annual Report: 1-26.

13. Zar-Kessler, C., Karaa, A., Sims, K. B., Clarke, V., \& Kuo, B. (2016). Understanding the gastrointestinal manifestations of Fabry disease: promoting prompt diagnosis. Therapeutic Advances in Gastroenterology, 9(4), 626-634

14. Pensabene, L., Sestito, S., Nicoletti, A., Graziano, F., Strisciuglio, P., \& Concolino, D. (2016). Gastrointestinal symptoms of patients with Fabry disease. Gastroenterology Research and Practice, 2016, 9712831

15. Marchesoni, C. L., Roa, N., Pardal, A. M., Neumann, P., Cáceres, G., Martínez, P., et al. (2010). Misdiagnosis in Fabry disease. Journal of Pediatrics, 156(5), 828-831

16. Replagal. Summary of Product Characteristics. Shire Pharmaceuticals Limited; 2021.

17. Fabrazyme. Prescribing information. Genzyme Corporation; 2018.

18. Galafold. Prescribing information. Amicus Therapeutics Inc; 2020.

19. Schiffmann, R., Hughes, D. A., Linthorst, G. E., Ortiz, A., Svarstad, E., Warnock, D. G., et al. (2017). Screening, diagnosis, and management of patients with Fabry disease: conclusions from a "Kidney Disease: Improving Global Outcomes" (KDIGO) Controversies Conference. Kidney International, 91(2), 284-293

20. Dehout, F., Roland, D., Treille de Granseigne, S., Guillaume, B., \& Van Maldergem, L. (2004). Relief of gastrointestinal symptoms under enzyme replacement therapy [corrected] in patients with Fabry disease. Journal of Inherited Metabolic Disease, 27(4), 499-505

21. Germain, D. P., Hughes, D. A., Nicholls, K., Bichet, D. G., Giugliani, R., Wilcox, W. R., et al. (2016). Treatment of Fabry's disease with the pharmacologic chaperone migalastat. New England Journal of Medicine, 375(6), 545-555

22. Wilcox, W. R., Feldt-Rasmussen, U., Martins, A. M., Ortiz, A., Lemay, R. M., Jovanovic, A., et al. (2018). Improvement of Fabry disease-related gastrointestinal symptoms in a significant proportion of female patients treated with agalsidase beta: data from the Fabry registry. JIMD Rep, 38, 45-51

23. Schiffmann, R., Bichet, D. G., Jovanovic, A., Hughes, D. A., Giugliani, R., Feldt-Rasmussen, U., et al. (2018). Migalastat improves diarrhea in patients with Fabry disease: clinical-biomarker 
correlations from the phase 3 FACETS trial. Orphanet Journal of Rare Diseases, 13(1), 68

24. Wraith, J. E., Tylki-Szymanska, A., Guffon, N., Lien, Y. H., Tsimaratos, M., Vellodi, A., et al. (2008). Safety and efficacy of enzyme replacement therapy with agalsidase beta: an international, open-label study in pediatric patients with Fabry disease. Journal of Pediatrics, 152(4), 563-570

25. Øvretveit, J., Zubkoff, L., Nelson, E. C., Frampton, S., Knudsen, J. L., \& Zimlichman, E. (2017). Using patient-reported outcome measurement to improve patient care. International Journal for Quality in Health Care, 29(6), 874-879

26. Armstrong, A. W., Banderas, B., Foley, C., Stokes, J., Sundaram, M., \& Shields, A. L. (2017). Development and psychometric evaluation of the self-assessment of psoriasis symptoms (SAPS) clinical trial and the SAPS - real world patient-reported outcomes. The Journal of Dermatological Treatment, 28(6), 505-514

27. Patrick, D. L., Burke, L. B., Gwaltney, C. J., Leidy, N. K., Martin, M. L., Molsen, E., et al. (2011). Content validity-establishing and reporting the evidence in newly developed patient-reported outcomes (PRO) instruments for medical product evaluation: ISPOR PRO Good Research Practices Task Force report: part 2-assessing respondent understanding. Value in Health, 14(8), 978-988

28. US Department of Health and Human Services FDA Center for Drug Evalution and Research, US Department of Health and Human Services FDA Center for Biologics Evaluation and Research, and US Department of Health and Human Services FDA Center for Devices and Radiological Health. Guidance for industry: patient-reported outcome measures: use in medical product development to support labeling claims: draft guidance (2006). Health Qual Life Outcomes, 4, 79
29. Wilson, I. B., \& Cleary, P. D. (1995). Linking clinical variables with health-related quality of life. A conceptual model of patient outcomes. JAMA, 273(1), 59-65

30. Svedlund, J., Sjodin, I., \& Dotevall, G. (1988). GSRS-a clinical rating scale for gastrointestinal symptoms in patients with irritable bowel syndrome and peptic ulcer disease. Digestive Diseases and Sciences, 33(2), 129-134

31. Drossman, D. A. (2006). The functional gastrointestinal disorders and the rome III process. Gastroenterology, 130, 1377-1390

32. Palsson, O. S., Whitehead, W. E., van Tilburg, M. A., Chang, L., Chey, W., Crowell, M. D., et al. (2016). Rome IV diagnostic questionnaires and tables for investigators and clinicians. Gastroenterology, S0016-5085(16), 00180-3. https://doi.org/10.1053/j. gastro.2016.02.014.

33. Noël, E., Dussol, B., Lacombe, D., Bedreddine, N., Fouilhoux, A., Ronco, P., et al. (2019). Treatment needs and expectations for Fabry disease in France: development of a new Patient Needs Questionnaire. Orphanet Journal of Rare Diseases, 14(1), 284

34. Kimonis, V., Frey, A., Politei, J., \& Üçeyler, N. (2020). A patientreported outcome validation study of concept elicitation and cognitive debriefing to understand neuropathic pain in Fabry disease. Molecular Genetics and Metabolism, 129(2), S89-S90

35. Turner-Bowker, D. M., Lamoureux, R. E., Stokes, J., LitcherKelly, L., Galipeau, N., Yaworsky, A., et al. (2018). Informing a priori sample size estimation in qualitative concept elicitation interview studies for clinical outcome assessment instrument development. Value in Health, 21(7), 839-842

Publisher's Note Springer Nature remains neutral with regard to jurisdictional claims in published maps and institutional affiliations. 